\title{
石墨烯负载离子液体的制备及其与六苯氧基 环三磷腈协效阻燃环氧树脂的性能研究
}

\author{
张凯伦, 陈伊阳, 姚猛, 王佳乐, 王爱卿, 屈红强", 徐建中 ${ }^{*}$ \\ 河北大学化学与环境科学学院, 河北省阻燃材料与加工技术工程研究中心, 保定 071002 \\ *通讯作者, E-mail: hqqu@163.com; xjz8112@126.com \\ 收稿日期: 2021-05-28; 接受日期: 2021-07-06; 网络版发表日期: 2021-09-06 \\ 京津冀协同创新建设项目(编号: 20541401D)和河北大学创新实验项目(编号: sy202045)资助
}

\begin{abstract}
摘要本文以可膨胀石墨(EG)和1-丁基-3-甲基咪唑六氟磷酸盐离子液体([BMIM] $\mathrm{PF}_{6}$ ) 为原料, 在去离子水中通 过绿色、简单的球磨法成功制备出了石墨烯负载离子液体杂化物(GnP@ILs), 并对其结构组成进行表征。将 GnP@ILs单独或与六苯氧基环三磷腈(HPCTP)混合加入到环氧树脂(EP)中，研究其对EP复合材料综合性能的影 响. 极限氧指数(LOI)、垂直燃烧(UL-94)和锥形量热测试结果表明, GnP@ILs能提高EP复合材料的阻燃性能，同 时与HPCTP复配的EP复合材料(EP/7.2 wt \%HPCTP $/ 1.8 \mathrm{wt} \% \mathrm{GnP} @ \mathrm{ILs})$ 的阻燃性能最好, LOI达到 $33.8 \%$, 并通过了 UL-94 V 0 级. EP/7.2wt\%HPCTP/1.8wt\%GnP@ILs的热释放速率峰值和总热释放量分别降低了 55.54\%和 $44.28 \%$. 同时, [BMIM] $\mathrm{PF}_{6}$ 的加入增强了阻燃剂与 $\mathrm{EP}$ 的界面相容性, $\mathrm{EP}$ 复合材料的拉伸强度和抗冲强度均明显提高.
\end{abstract}

关键词＼cjkstart石墨烯负载离子液体, 六苯氧基环三磷腈, 湿法球磨, 环氧树脂, 阻燃

\section{1 引言}

环氧树脂(EP)具有热稳定性高、力学性能好、绝 缘性好、耐化学腐蚀等优异性能, 被广泛应用于电子/ 电气绝缘、钢管、地坪、胶黏剂和涂料等领域 ${ }^{[1,2]}$.

然而，纯EP的高可燃性及在燃烧过程中会产生大量的 热量和烟雾限制了其应用. 因此，对EP进行阻燃处理 十分重要. 传统的阻燃剂主要为含溴阻燃剂或者无机 化合物阻燃剂，然而含溴阻燃剂燃烧过程中会产生一 些有毒有害的物质，如二恶英，危害人体健康 ${ }^{[3]}$; 无机 化合物添加量大, 与聚合物相容性差, 降低了聚合物的
力学性能 ${ }^{[4]}$. 因此, 开发一种新型高效的阻燃剂十分 必要.

石墨烯是由碳原子构成的二维片层结构，该结构 使得石墨烯在阻燃聚合物应用中具有很大的发展潜 能. 其阻燃机理可概述为: 在燃烧过程中石墨烯会形成 致密且连续的炭层, 对基体起到保护作用, 阻止外界氧 气的进入 ${ }^{[5]}$; 石墨烯良好的导热性会转移燃烧过程中 局部过多的热量, 从而抑制火势的传播 ${ }^{[6]}$; 良好的导热 和炭层的阻隔作用产生了所谓的“迷宫效应”，使得热 量和气体通向聚合物基体的路径“曲折”，有效防止了 火焰的蔓延 ${ }^{[7]}$. 然而, 石墨烯片层之间存在较强的范德

引用格式: Zhang K, Chen Y, Yao M, Wang J, Wang A, Qu H, Xu J. The preparation of ionic liquid doped graphene composite and its synergistic flame retardant effect with hexaphenoxy cyclotriphosphazene on epoxy resin. Sci Sin Chim, 2021, 51: 1283-1292, doi: 10.1360/SSC-2021-0129 
华力，在加工应用的过程中石墨烯容易发生团聚从而 影响材料的综合性能 ${ }^{[8]}$. 因此，通过引入阻燃分子(含 金属、含磷、含嗍和含氮化合物)替换石墨烯片层表 面和边缘的含氧官能团来对其进行改性处理，可增加 其分散性或减弱片层之间的相互作用，逐渐发展成为 一种改善高分子材料综合性能的有效方式 ${ }^{[9]}$.

离子液体(ILs)作为一种“绿色”溶剂，因其极低的 挥发性和低可燃性等一些独特的性能而被广泛应用到 很多领域 ${ }^{[10]}$. 研究表明, ILs在阻燃领域中也有独特的 作用. 例如, $\mathrm{Li}^{\text {等 }}{ }^{[11]}$ 合成了一种非共价离子液体阻燃剂 功能化的氮化嗍纳米片(ILFR-fBNNSs)，并将其加入 EP中来研究其对EP材料的综合性能的影响. 由于其独 特的结构, EP复合材料的导热性能和阻燃性能得到了 很大提升, 热释放速率峰值(PHRR)和总热释放量 (THR)分别下降了 $42.4 \%$ 和 $37.7 \% . \mathrm{Wu}^{\text {等 }}{ }^{[12]}$ 合成了一种 新型多功能离子液体基金属-有机杂化物(PMAIL), 发 现在 EP 中添加量为 $6 \mathrm{wt} \%$ 时即可通过垂直燃烧 (UL-94) $\mathrm{V} 0$ 级. Wang 等 ${ }^{[13]}$ 设计了一种新型含磷无卤的 离子液体并将其添加到EP中，结果发现仅添加量为 $4 \mathrm{wt} \%$ 时可通过UL-94 V 0 级, 极限氧指数(LOI)由 $26.5 \%$ 提升到 $32.5 \%$. 其中, 1-丁基-3-甲基咪唑六氟磷酸盐离子 液体 $\left([\mathrm{BMIM}] \mathrm{PF}_{6}\right.$ ) 不仅含有 $\mathrm{N} 、 \mathrm{P}$ 和 $\mathrm{F}$ 等阻燃元素，并且 由于 $[\mathrm{BMIM}] \mathrm{PF}_{6}$ 的表面张力与石墨的表面能十分匹配, 所以剥离石墨的效率会在一定程度上有所提高 ${ }^{[14]}$.

在本工作中, 我们以可膨胀石墨(EG)和[BMIM]$\mathrm{PF}_{6}$ 为原料, 在去离子水中通过绿色、简单的球磨法成
功制备出了石墨烯负载离子液体的杂化物(GnP@ILs), 随后将其单独或与六苯氧基环三磷腈(HPCTP)混合加 入到EP中来提高EP复合材料的阻燃性能和力学性能, 并对阻然机理进行了分析.

\section{2 实验部分}

\section{1 材料}

EG (平均粒径: $178 \mu \mathrm{m}$, 膨胀倍数: 300 )于青岛沿 海碳素材料有限公司购买(中国); [BMIM] $\mathrm{PF}_{6}$ 由兰州 中科凯特科工贸有限公司供应(中国); HPCTP由日本 大冢化学提供; EP(E44)由中国石化集团公司资产管 理公司巴陵石化分公司提供(中国); 间苯二胺 $(m-\mathrm{PDA})$ 购自阿拉丁工业有限公司(中国).

\subsection{GnP@ILs的制备}

利用 $\mathrm{EG}$ 与 $[\mathrm{BMIM}] \mathrm{PF}_{6}$ 之间的 $\pi-\pi$ 共轭作用, 通过湿 式球磨法成功制备了 $\mathrm{GnP} @ \mathrm{IL}$. 将EG和[BMIM] $\mathrm{PF}_{6}$ 按 $4: 1$ 的比例混合, 再将其与水混合(水和混合物的比例 为 $10: 1$ )超声 $30 \mathrm{~min}$ 后放入盛有氧化锆的球磨罐中, 用 湿法球磨机在 $2500 \mathrm{r} / \mathrm{min}$ 下球磨 $40 \mathrm{~h}$. 通过球磨珠在高 速转动下产生的机械力将石墨片成功剥离并且将 $[\mathrm{BMIM}] \mathrm{PF}_{6}$ 成功负载到石墨片层表面从而制得较少层 的GnP@ILs. 分别取10、20、30和40 h 的产物进行烘 干得到黑色粉末，取产物进行一系列表征来确定最佳 球磨时间.

表 1 EP复合材料样品成分

Table 1 Composition of EP composite samples

\begin{tabular}{|c|c|c|c|c|}
\hline \multirow{2}{*}{ 样品 } & \multicolumn{4}{|c|}{ 样品组分 $(w t \%)$} \\
\hline & Resin & НРCTP & GnP@ILs & $\mathrm{GnP}$ \\
\hline Pure EP & 100 & 0 & 0 & 0 \\
\hline EP/3wt\%GnP@ILs & 100 & 0 & 3 & 0 \\
\hline EP/6wt\%GnP@ILs & 100 & 0 & 6 & 0 \\
\hline EP/9wt\%GnP@ILs & 100 & 0 & 9 & 0 \\
\hline $\mathrm{EP} / 9 \mathrm{wt} \% \mathrm{GnP}$ & 100 & 0 & 0 & 9 \\
\hline $\mathrm{EP} / 9 \mathrm{wt} \% \mathrm{HPCTP}$ & 100 & 9 & 0 & 0 \\
\hline EP/8.1wt $\%$ HPCTP $/ 0.9 w t \% G n P @ I L s$ & 100 & 8.1 & 0.9 & 0 \\
\hline EP/7.2wt $\%$ HPCTP/1.8wt\%GnP@ILs & 100 & 7.2 & 1.8 & 0 \\
\hline EP/6.3wt\%HPCTP/1.7wt\%GnP@ILs & 100 & 6.3 & 2.7 & 0 \\
\hline
\end{tabular}




\subsection{EP/GnP@ILs及EP/HPCTP/GnP@ILs复合材 料的制备}

表1列出了EP复合材料中阻燃剂的含量配方，选 取 $\mathrm{GnP} @ I L s$ 含量为 $9 \mathrm{wt} \%$ 的EP复合材料作为典型制备 工艺. 表1中的纯EP、不同含量的EP/GnP@ILs和EP/ HPCTP/GnP@ILs复合材料的制备工艺除阻燃剂含量 不同外，制备工艺类似。具体如下：首先将 $11.7 \mathrm{~g}$ GnP@ILs倒入 $130.0 \mathrm{~g} \mathrm{EP}$ 中, 在 $60^{\circ} \mathrm{C}$ 的油浴加热下磁 摚拌 $20 \mathrm{~min}$; 然后向混合物中加入 $14.3 \mathrm{~g} \mathrm{~m}$-PDA并摚 拌 $20 \mathrm{~min}$; 最后将混合物倒入模具中进行固化, 固化 后, 让样品冷却至室温. 固化条件为: 首先在 $60^{\circ} \mathrm{C}$ 的真 空烘箱中固化 $20 \mathrm{~min}$, 然后在 $80^{\circ} \mathrm{C}$ 的吹风烘箱中固化 $2 \mathrm{~h}$, 最后在 $150^{\circ} \mathrm{C}$ 下固化 $3 \mathrm{~h} 40 \mathrm{~min}$.

\section{4 表征}

在所有样品进行喷金处理后采用JSM-7500F扫描 电子显微镜(SEM, JEOL, 日本)和能谱仪(EDS)对其进 行表面形貌表征和元素组成分析; 将样品在无水乙醇 中超声分散, 然后放入铜栅中, 利用 $\mathrm{G} 2 \mathrm{~F} 20$ 透射电子 显微镜(TEM, S-TWIN, 美国)在 $200 \mathrm{kV}$ 的加速电压下 观察样品的结构形貌; 晶体结构由D8-ADVANCE X射 线衍射光谱仪(XRD, Bruker, 德国)在辐射管的电流和 电压分别为 $40 \mathrm{~mA}$ 和 $40 \mathrm{kV}$ 的条件下进行测量; 样品的 元素组成及化学态分析由PHI 5300 ESCA X射线光电 子能谱仪(XPS, PE, 美国)在低于 $10^{-6} \mathrm{~Pa}$ 的真空下进行 测量; GnP@ILs的片层厚度由Solver P47原子力显微镜 (AFM，NT-MDT，俄罗斯)进行测量; 拉曼光谱由反射 共焦激光拉曼光谱仪(HORIBA, JobinYvon Ltd, 法国) 在室温下获得; 所有样品的热重分析(TGA)数据都是

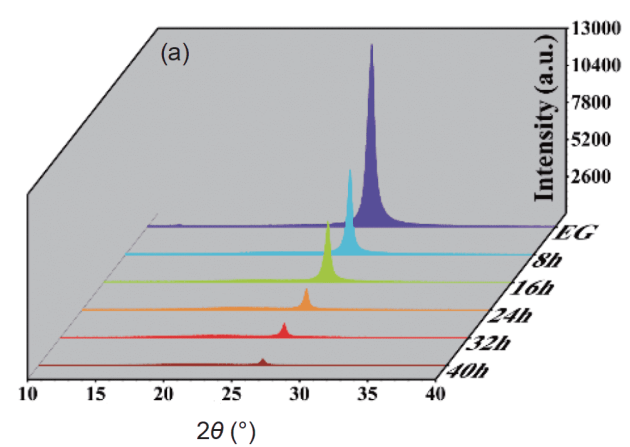

使用SAT 449C热分析仪(TGA, NETZSCH, 德国)进行 测量; 将尺寸为 $130 \mathrm{~mm} \times 13 \mathrm{~mm} \times 3.2 \mathrm{~mm}$ 的样品根据 ANSI/UL-94标准, 用PX-03-001垂直燃烧仪器(UL-94, PHINIX, 中国)进行测量; 将尺寸为 $130 \mathrm{~mm} \times$ $6.5 \mathrm{~mm} \times 3 \mathrm{~mm}$ 的样品根据ASTM D2863标准，用JF-3 型氧指数仪 (LOI, 江宁分析仪器公司, 中国)进行测量; 将尺寸为 $100 \mathrm{~mm} \times 100 \mathrm{~mm} \times 3 \mathrm{~mm}$ 的样品按ISO5660标 准, 用iCone Plus雉形量热仪(Cone, FTT, 英国)在 $50 \mathrm{kw} / \mathrm{m}^{2}$ 的外热流下用铝䈃纸包裹进行测量.

\section{3 结果与讨论}

\subsection{GnP@ILs的结构及组成}

为了证明得到的产物为 GnP @ILs, 利用 TEM、 XRD、XPS、AFM、拉曼光谱和傅里叶转换红外光 谱(FTIR)对其进行表征. 图1为GnP@ILs的XRD和拉曼 图像. 从图 $1 \mathrm{a}$ 中可以看出, $0 \sim 40 \mathrm{~h}$ 内的球磨产物在 $26.5^{\circ}$ 附近产生的对应于石墨002晶面结构的特征衍射峰在 逐步降低, 峰宽在逐渐增加, 这说明球磨的机械力成功 地对石墨层进行了剥离 ${ }^{[15]}$. 然而, 在 $26.5^{\circ}$ 附近区域的 特征衍射峰并没有消失, 说明没有剥离成单片层的石 墨烯. 图1b中, EG的拉曼光谱显示出 3 个峰: $1355 \mathrm{~cm}^{-1}$ 的D带峰, 代表 $\mathrm{C}$ 原子晶格的缺陷, $1580 \mathrm{~cm}^{-1}$ 的 $\mathrm{G}$ 带峰, 归因于 $\mathrm{C}$ 原子 $\mathrm{sp}^{2}$ 杂化的面内伸缩振动, $2700 \mathrm{~cm}^{-1}$ 的 $2 \mathrm{D}$ 带峰, 这是 $\mathrm{D}$ 峰的二阶模式 ${ }^{[16]}$. 经过机械剥离后 GnP@ILs的拉曼光谱D、G和2D带峰略微偏移 ${ }^{[17]}$, 位 于 1359,1570 和 $2695 \mathrm{~cm}^{-1}$ 处. GnP@ILs的 $I_{\mathrm{D}} / I_{\mathrm{G}}$ 值为 0.51 大于 $\mathrm{EG}$ 的 $I_{\mathrm{D}} / I_{\mathrm{G}}$ 值 $(0.31)$, 表明 GnP@ILs存在着边缘 缺陷特征 ${ }^{[18,19]}$.

图2为GnP@ILs的TEM和元素分布图. 从图2a中

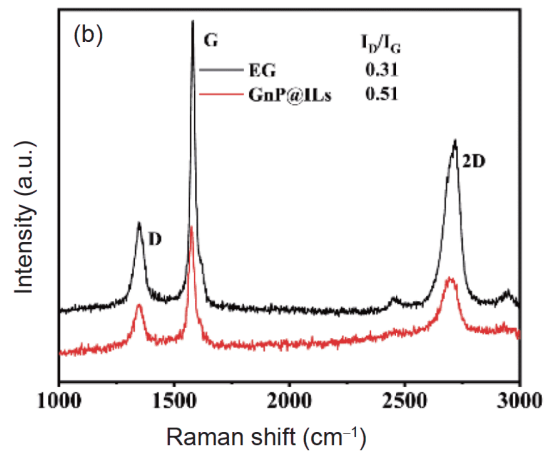

图 1 球磨产物0 40 h的XRD (a), EG和GnP@ILs的拉曼光谱(b) (网络版彩图)

Figure 1 XRD image of ball milled product at 0-40 h (a), and Raman spectra of EG and GnP@ILs (b) (color online). 


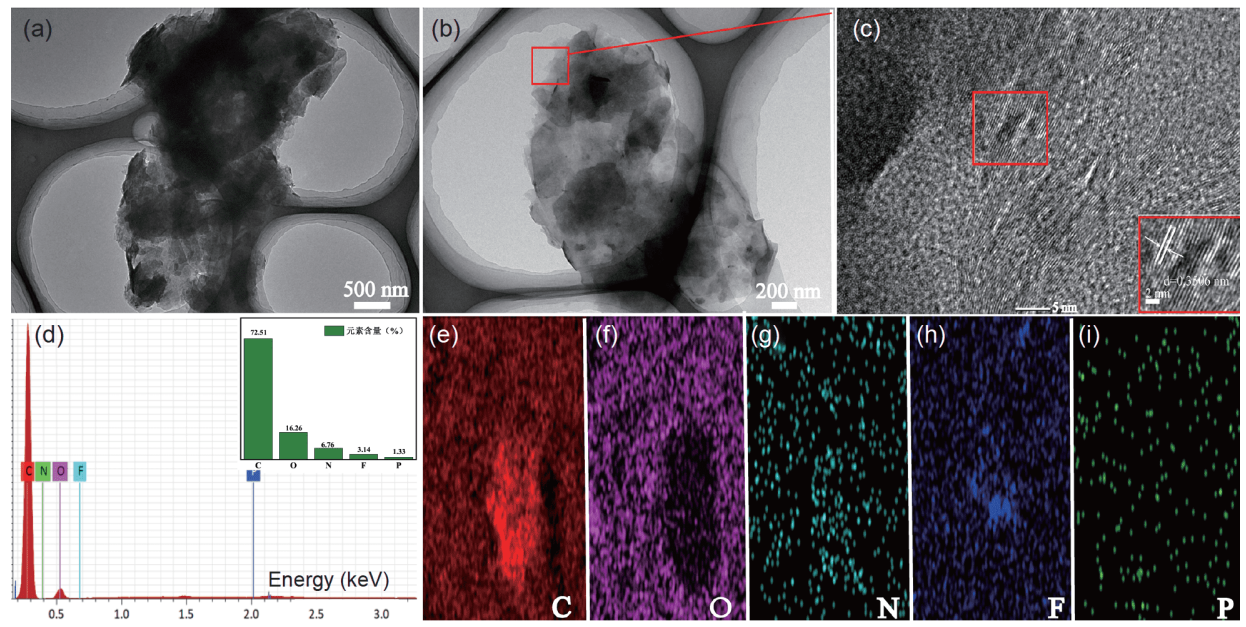

图 2 (a c) GnP@ILs的TEM图; (d i) 选区能谱和元素分布图(网络版彩图)

Figure 2 (a-c) TEM images of GnP@ILs; (d-i) the mapping images of GnP@ILs (color online).
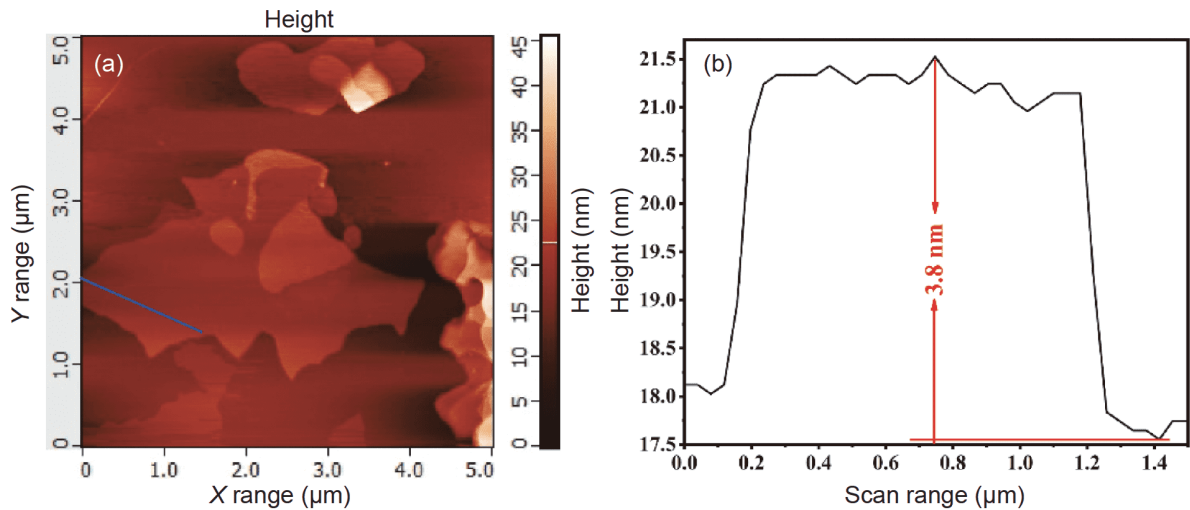

图 3 GnP@ILs的AFM图(网络版彩图)

Figure 3 AFM image of GnP@ILs (color online).

可以看出，球磨40h后GnP@ILs的TEM图像呈现横向 尺寸约为 $2 \mu \mathrm{m}$ 的皱缩半透明纳米片，在高倍率下对 GnP@ILs边缘晶格条纹进行分析，发现GnP@ILs的晶 格间距 $d=0.3506 \mathrm{~nm}$ (图2b, c), 初步推测生成了多层石 墨烯纳米片．通过图2d i 所示区域的EDS元素分布图 可以观察到GnP@ILs上有 $C 、 O 、 N 、 F$ 和P原子，且 $F$ 元素含量为 $3.14 \%$, 证明了 $\left[\mathrm{BMIM} \mathrm{PF}_{6}\right.$ 成功负载到石 墨烯片层上. 同时，又通过AFM来测量GnP@ILs的厚 度, 如图3所示. GnP@ILs表现出超薄纳米片的形式, 直径约几微米, 厚 $3.8 \mathrm{~nm}$ 左右, 由于石单层墨烯厚度约 为 $0.34 \mathrm{~nm}^{[20]}$, 石墨烯杂化物为 $0.34 \sim 1 \mathrm{~nm}$ 不等, 这说明 了产物是由多层石墨烯堆叠组成.

通过FTIR和XPS来对GnP@ILs的官能团和化学成 键方式进行分析，如图4和5所示. 对比图4中EG和

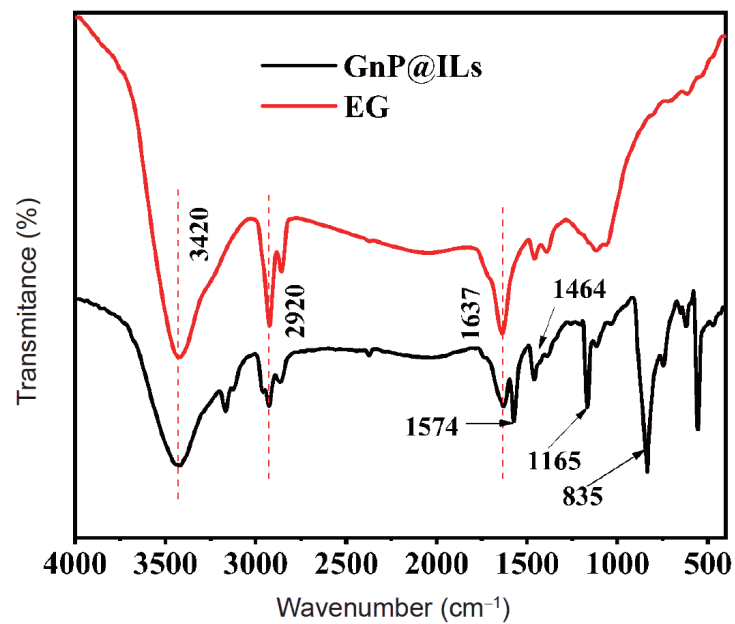

图 4 EG和GnP@ILs的FTIR图(网络版彩图)

Figure 4 FTIR image of EG and GnP@ILs (color online). 
$\mathrm{GnP} @ \mathrm{ILs}$ 的FTIR图, 发现 $3420 \mathrm{~cm}^{-1}$ 为羟基的拉伸振动 峰; 2920 和 $1637 \mathrm{~cm}^{-1}$ 出现的峰分别归因于 $-\mathrm{CH}_{2}-$ 和 $\mathrm{C}=\mathrm{O}$ 的振动. 此外, 1574、1464和 $1165 \mathrm{~cm}^{-1}$ 附近出现 的峰是由咪唑环骨架振动引起的; $1000 \mathrm{~cm}^{-1}$ 以下出现 的峰为各种 $\mathrm{C}-\mathrm{H}$ 面内弯曲振动引起的, 并且在 $835 \mathrm{~cm}^{-1}$ 处出现了 P-F特征吸收峰. 从 GnP @ILs的XPS (图5a)中观察到, 在 $686 \mathrm{eV}$ 出现了 $\mathrm{F} 1 \mathrm{~s}$ 峰, 在 533 和 $285 \mathrm{eV}$ 附近出现了 $\mathrm{O} 1 \mathrm{~s}$ 和 $\mathrm{C} 1 \mathrm{~s}$ 峰; 分别从 $\mathrm{GnP} @ \mathrm{ILs}$ 的 $\mathrm{C} 1 \mathrm{~s} 、 \mathrm{O} 1 \mathrm{~s}$ 和 $\mathrm{F} 1 \mathrm{~s}$ 进行拟合后的高分辨图谱(图 $5 \mathrm{~b} \sim \mathrm{d}$ )中 可以发现，在 291.5 和 $288.0 \mathrm{eV}$ 处的峰分别归属于C-F 和 $\mathrm{N}-\mathrm{C}-\mathrm{N}$ 的结合能 ${ }^{[21,22]}$, 在 535.0 和 $533.9 \mathrm{eV}$ 处的峰分 别对应于 $\mathrm{O}-\mathrm{F}$ 和 $\mathrm{C}-\mathrm{O}-\mathrm{P}$ 的结合能 ${ }^{[22,23]}$, 在 688.4 和 $686.4 \mathrm{eV}$ 处的峰分别是 $\mathrm{C}-\mathrm{F}$ 和 $\mathrm{P}-\mathrm{F}$ 键所对应的结合 能 ${ }^{[22]}$. GnP@ILs的FTIR 和XPS结果均说明[BMIM]PF 成功地负载到石墨烯片层上.

\section{2 阻燃性能}

\subsubsection{LOI和UL-94分析}

通常情况下用LOI和UL-94来量化材料的阻燃性 能. 当LOI值高且UL-94通过V 0 等级时, 材料表现出较
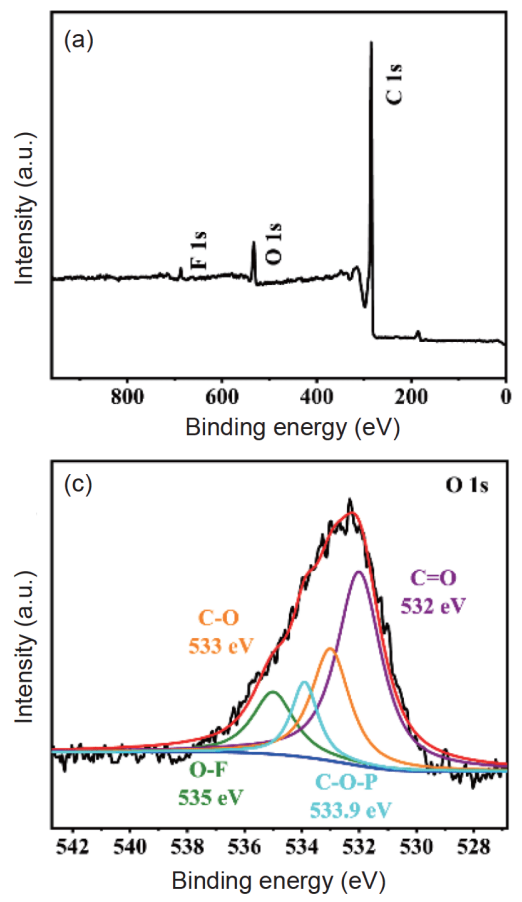

好的阻燃性能. EP及其复合材料的LOI和UL-94测试 结果如表2 所示. 从表中可以看出, 纯EP的LOI值为 $23.5 \%$. 在只添加阻燃剂GnP@ILs的EP复合材料中, 随着GnP@ILs添加量的增加, EP复合材料的LOI值逐 渐增加, EP $/ 9 \mathrm{wt} \% \mathrm{GnP} @ \mathrm{ILs}$ 表现出最高的LOI值为 $27.5 \%$ ，明显高于 $\mathrm{EP} / 9 \mathrm{wt} \% \mathrm{GnP}$ (LOI为 $26.1 \%$ ), 但 UL-94仍没有等级. 因此，选择GnP@ILs与HHPCP协 效来进一步提高EP的阻燃性能. 以 $9 \mathrm{wt} \%$ 的添加量为 纵向对比点, 可以看出, 随着 $\mathrm{GnP} @$ ILs在HPCTP中比 例的增加，阻燃性能先增加后降低，EP/8.1wt \% HPCTP $/ 0.9 w t \% G n P @ I L s$ 和EP $/ 7.2 w t \% H P C T P / 1.8 w t \%$ GnP@ILs的UL-94等级均达到V 0级，LOI值分别为 $33.0 \%$ 和 $33.8 \%$. 结果表明, GnP@ILs的加入能提高EP 的阻燃性能, 同时与HPCTP存在协效作用, 可提高EP 的阻燃性能.

\subsection{2 雉形量热测试分析}

雉形量热测试(CCT)被认为是实验室模拟测试材 料燃烧性能的有力工具, 经过LOI和UL-94对EP复合材 料阻燃性能的初步篮选，选取阻燃剂添加量为 $9 \mathrm{wt} \%$ 的GnP@ILs、9 wt \% 的HPCTP和 $7.2 \mathrm{wt} \% \mathrm{HPCTP} / 1.8 \mathrm{wt} \%$
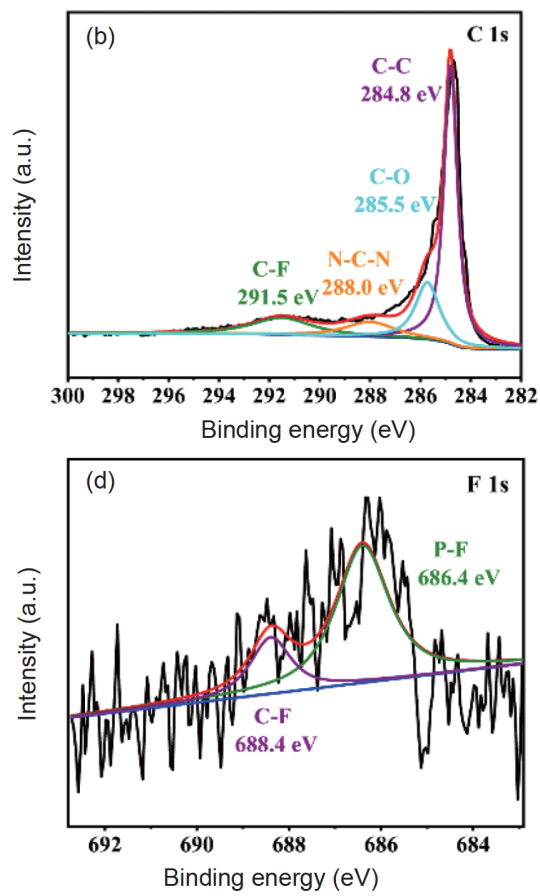

图 5 (a) GnP@ILs的XPS图; (b d) GnP@ILs的C1s、O1s和F1s高分辨率XPS图(网络版彩图)

Figure 5 (a) XPS image of GnP@ILs; (b-d) high-resolution XPS image of C1s, O1s and F1s of GnP@ILs (color online). 
表 2 EP及其复合材料的LOI和UL-94测试数据

Table 2 LOI and UL-94 test data of EP composites

\begin{tabular}{ccccc}
\hline 样品 & LOI $(\%)$ & & UL-94 & Rating \\
\cline { 3 - 5 } Pure EP & 23.5 & $t 1(\mathrm{~s})$ & - & No rating \\
EP/3wt\%GnP@ILs & 26.3 & - & - & No rating \\
EP/6wt\%GnP@ILs & 27.2 & - & - & No rating \\
EP/9wt\%GnP@ILs & 27.5 & - & - & No rating \\
EP/9wt\%GnP & 26.1 & - & - & No rating \\
EP/9wt\%HPCTP & 31.3 & 15.1 & 6.5 & V 1 \\
EP/8.1wt\%HPCTP/0.9wt\%GnP@ILs & 33.0 & 9.9 & 5.6 & V 0 \\
EP/7.2wt\%HPCTP/1.8wt\%GnP@ILs & 33.8 & 7.6 & 4.0 & V 0 \\
EP/6.3wt\%HPCTP/2.7wt\%GnP@ILs & 32.7 & 11.1 & 6.0 & V 1 \\
\hline
\end{tabular}

GnP@ILs进行CCT分析, 结果如图6和表3所示. 图6分 别给出了 $\mathrm{EP}$ 及其复合材料的热释放速率 (HRR)、 THR、烟释放速率峰值(PSPR) 和总烟释放量(TSP) 随 时间变化的曲线, 表3列出了点燃时间(TTI)、PHRR和 平均有效燃烧热(av-EHC)等重要数据.

PHRR是评价火灾性能的重要参数. 较高的PHRR 值反映了较低的阻燃性 ${ }^{[24]}$. 从图6a中可以看出, 纯 $\mathrm{EP}$ 在点火 $59 \mathrm{~s}$ 后迅速燃烧，PHRR值达到了 $1093 \mathrm{kw} / \mathrm{m}^{2}$. 而在加入 GnP@ILs和HPCTP后, EP复合材料的TTI有
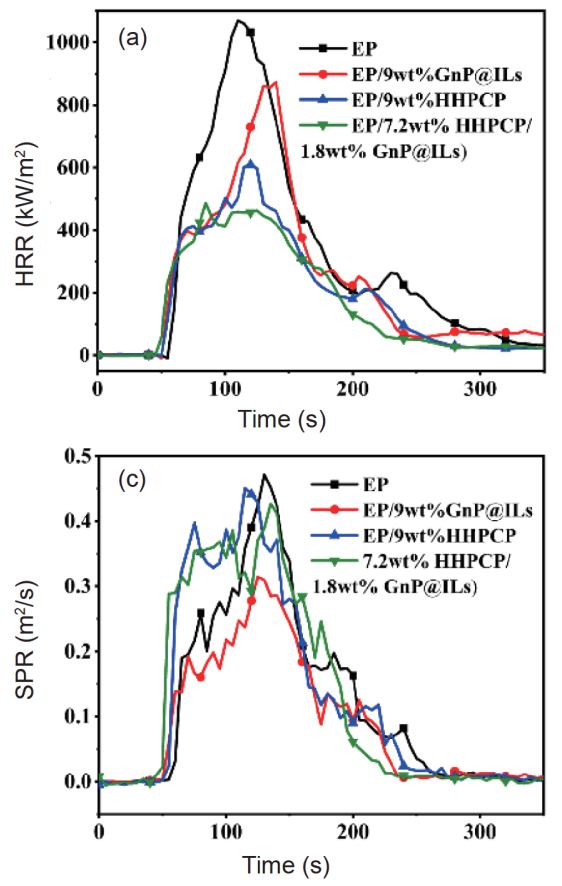

所提前, 这可能是因为离子液体和膦腈基团的引入促 进了EP基体的提前分解. 虽然加入阻燃剂的EP复合材 料TTI有所提前, 但是其PHRR得到了明显的降低. EP/ $9 \mathrm{wt} \% \mathrm{GnP} @ \mathrm{ILs}$ 的PHRR为 $872 \mathrm{kw} / \mathrm{m}^{2}$, 降低了 $20.22 \%$. 同时, 随着HPCTP和GnP@ILs复配加入, EP/9wt \% HPCTP和EP/7.2wt $\%$ HPCTP/1.8wt\%GnP@ILs的PHRR 分别为 608 和 $486 \mathrm{kw} / \mathrm{m}^{2}$, 分别降低了 $44.37 \%$ 和 $55.54 \%$. 表明HPCTP和GnP@ILs间存在协效作用，两者共同作 用可提高EP材料的阻燃性能.
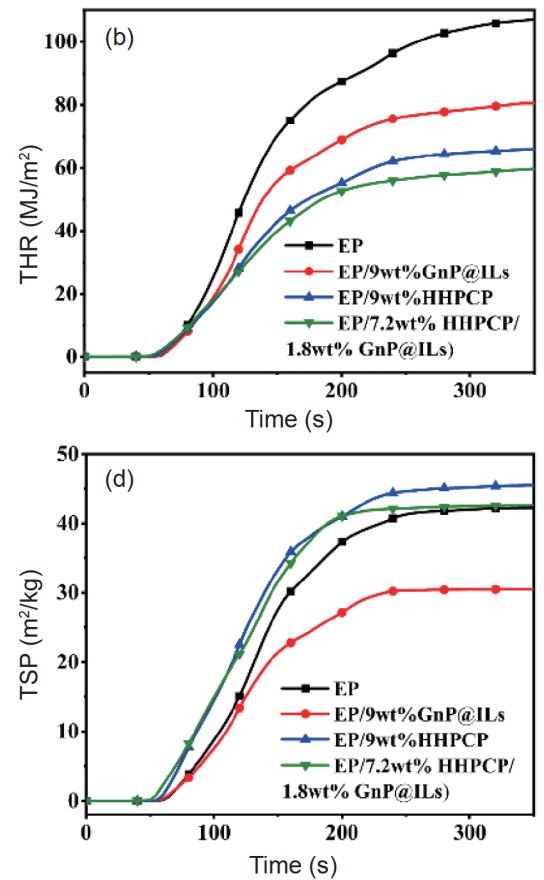

图 6 EP复合材料的HRR (a)、THR (b)、SPR (c)和TSP (d)图(网络版彩图)

Figure 6 HRR (a), THR (b), SPR (c) and TSP (d) diagrams of EP composites (color online). 
表 $3 \mathrm{EP}$ 及其复合材料的 CCT数据

Table 3 CCT data of EP and its composite materials

\begin{tabular}{ccccccc}
\hline 样品 & TTI $(\mathrm{S})$ & PHRR $\left(\mathrm{kW} / \mathrm{m}^{2}\right)$ & $\mathrm{THR}\left(\mathrm{MJ} / \mathrm{m}^{2}\right)$ & $\mathrm{PSPR}\left(\mathrm{m}^{2} / \mathrm{s}\right)$ & $\mathrm{TSP}\left(\mathrm{m}^{2} / \mathrm{kg}\right)$ & $\mathrm{Av}-\mathrm{EHC}(\mathrm{MJ} / \mathrm{kg})$ \\
\hline Pure EP & 59 & 1093 & 107.03 & 0.47 & 42.21 & 26.62 \\
EP/9wt\%GnP@ILs & 57 & 872 & 80.50 & 0.31 & 30.51 & 23.02 \\
EP/9wt\%HPCTP & 54 & 608 & 66.01 & 0.45 & 45.71 & 17.06 \\
EP/7.2wt\%HPCTP/1.8wt\%GnP@ILs & 56 & 486 & 59.64 & 0.42 & 42.59 & 16.60 \\
\hline
\end{tabular}

THR曲线的梯度是衡量火焰传播速度的重要指 标. 从图 $6 \mathrm{~b}$ 中可以看出, 纯 $\mathrm{EP}$ 的 THR为 $107.03 \mathrm{MJ} / \mathrm{m}^{2}$, 而添加阻燃剂的EP复合材料的THR值均明显降低. $\mathrm{EP} / 9 \mathrm{wt} \% \mathrm{GnP} @ \mathrm{ILs}$ 的THR为 $80.50 \mathrm{MJ} / \mathrm{m}^{2}$, 降低了 $24.79 \%$, 而随着HPCTP和GnP@ILs的复配加入, EP/ $9 \mathrm{wt} \% \mathrm{HPCTP}$ 和EP/7.2wt $\% \mathrm{HPCTP} / 1.8 \mathrm{wt} \% \mathrm{GnP} @ \mathrm{ILs}$ 的 THR分别为 66.01 和 $59.64 \mathrm{MJ} / \mathrm{m}^{2}$, 降低了 $38.33 \%$ 和 $44.28 \%$. EP复合材料的PHRR和THR降低可归因于两 方面. 一方面由于 GnP@ILs的加入增加了阻燃剂与EP 基体的相容性，有利于阻燃剂的均匀分散 ${ }^{[25]}$; 同时, GnP@ILs在燃烧过程中形成致密的炭层, 产生了所谓 的“迷宫效应”，使热量和燃烧气体通向聚合物基体的 路径“曲折”，从而延缓了可燃气体与聚合物之间的直 接接触 ${ }^{[26]}$. 另一方面, 由于HPCTP的加入会产生大量 难燃气体如 $\mathrm{NH}_{3}$ 和水蒸气等对可燃性气体进行稀释, 从而减缓复合材料的剧烈燃烧; 并且HPCTP中含较多 的磷，会在凝聚相中产生磷酸、焦磷酸等物质促进残 炭的生成 ${ }^{[27]}$.

实际生活中，烟雾是火灾营救过程中的一个重要 危害, 降低烟雾的产生量至关重要 ${ }^{[28]}$. 从图6c、d中可 以看出, 纯EP在燃烧过程中会产生大量的烟雾, PSPR 和TSP分别为 $0.47 \mathrm{~m}^{2} / \mathrm{s}$ 和 $42.21 \mathrm{~m}^{2} / \mathrm{kg}$, 而在加入 $\mathrm{GnP} @$ ILs后EP复合材料的PSPR和TSP明显降低, EP/ $9 \mathrm{wt} \% \mathrm{GnP} @ \mathrm{ILs}$ 的效果最佳, PSPR和TSP分别为 $0.31 \mathrm{~m}^{2} / \mathrm{s}$ 和 $30.51 \mathrm{~m}^{2} / \mathrm{kg}$, 降低了 $34.04 \%$ 和 $27.72 \%$, 这是
由于 GnP@ILs的加入改善了阻燃剂与EP基体的界面相 容性, 从而增强了残炭的稳定性, 阻碍了基体的进一步 燃烧并且增加了烟雾吸收效果 ${ }^{[25]}$. 然而, 在加入 HPCTP 和GnP@ILs的混合物后，抑烟效果不明显甚至烟雾有 所上升, 主要是因为HPCTP会增加碳化反应, 并且某些 碳化产物会挥发到气相, 形成烟雾增加烟气密度.

av-EHC被用于分析阻燃剂气相作用机理. 较低的 值表示存在不可燃气体. 在表3中, EP/9wt\%GnP@ILs 的av-EHC从 $26.62 \mathrm{MJ} / \mathrm{kg}$ (纯EP)降低至 $23.02 \mathrm{MJ} / \mathrm{kg}$, 降 低程度较小. 可以认为, 添加 GnP@ILs 可以改善阻燃剂 与EP基体的界面相容性, 从而降低燃烧程度. 而随着 GnP@ILs和HPCTP的混合加入, EP/7.2wt $\%$ HPCTP/ $1.8 \mathrm{wt} \% \mathrm{GnP} @ \mathrm{ILs}$ 的av-EHC从 $26.62 \mathrm{MJ} / \mathrm{kg}$ 降至 $16.60 \mathrm{MJ} / \mathrm{kg}$. 这可以归因于两个方面. 一方面, HPCTP 被热分解形成PO', 它与 EP分解产生的高能自由基 $\mathrm{H}$ ·结合以减慢燃烧程度, 并且HPCTP会释放出不可燃 气体, 如挥发性磷化物、 $\mathrm{NH}_{3}$ 和水蒸气等 ${ }^{[27]}$. 这些气体 可以稀释可燃气体, 并降低EP复合材料周围的 $\mathrm{O}_{2}$ 浓度; 另一方面, GnP@ILs可以改善阻燃剂与EP基体的界面 相容性从而降低燃烧程度. 因此, GnP@ILs和HPCTP 起着协同作用, 可阻止火势蔓延并降低火灾危险.

\section{$3.3 \mathrm{EP}$ 复合材料的力学性能}

表4列出了EP及其复合材料的拉伸强度(TS)、断 裂伸长率 $(\mathrm{EB})$ 和冲击强度的数据, 所有数据均是由 5 根

表 4 EP及其复合材料的力学性能

Table 4 Mechanical properties of EP and its composite materials

\begin{tabular}{cccc}
\hline Sample & TS $(\mathrm{MPa})$ & EB $(\%)$ & Fracture toughness $\left(\mathrm{kJ} / \mathrm{m}^{2}\right)$ \\
\hline Pure EP & $48.96 \pm 0.35$ & $8.96 \pm 0.35$ & $7.49 \pm 0.31$ \\
EP/9wt\%GnP@ILs & $47.93 \pm 0.86$ & $9.01 \pm 0.26$ & $8.10 \pm 0.21$ \\
EP/9wt\%GnP & $30.66 \pm 0.45$ & $6.32 \pm 0.11$ & $6.12 \pm 0.16$ \\
EP/9wt\%HPCTP & $28.16 \pm 0.26$ & $5.96 \pm 0.16$ & $5.53 \pm 0.13$ \\
EP/7.2wt\%HPCTP/1.8wt\%GnP@ILs & $47.83 \pm 0.63$ & $8.56 \pm 0.23$ & $7.02 \pm 0.23$ \\
\hline
\end{tabular}




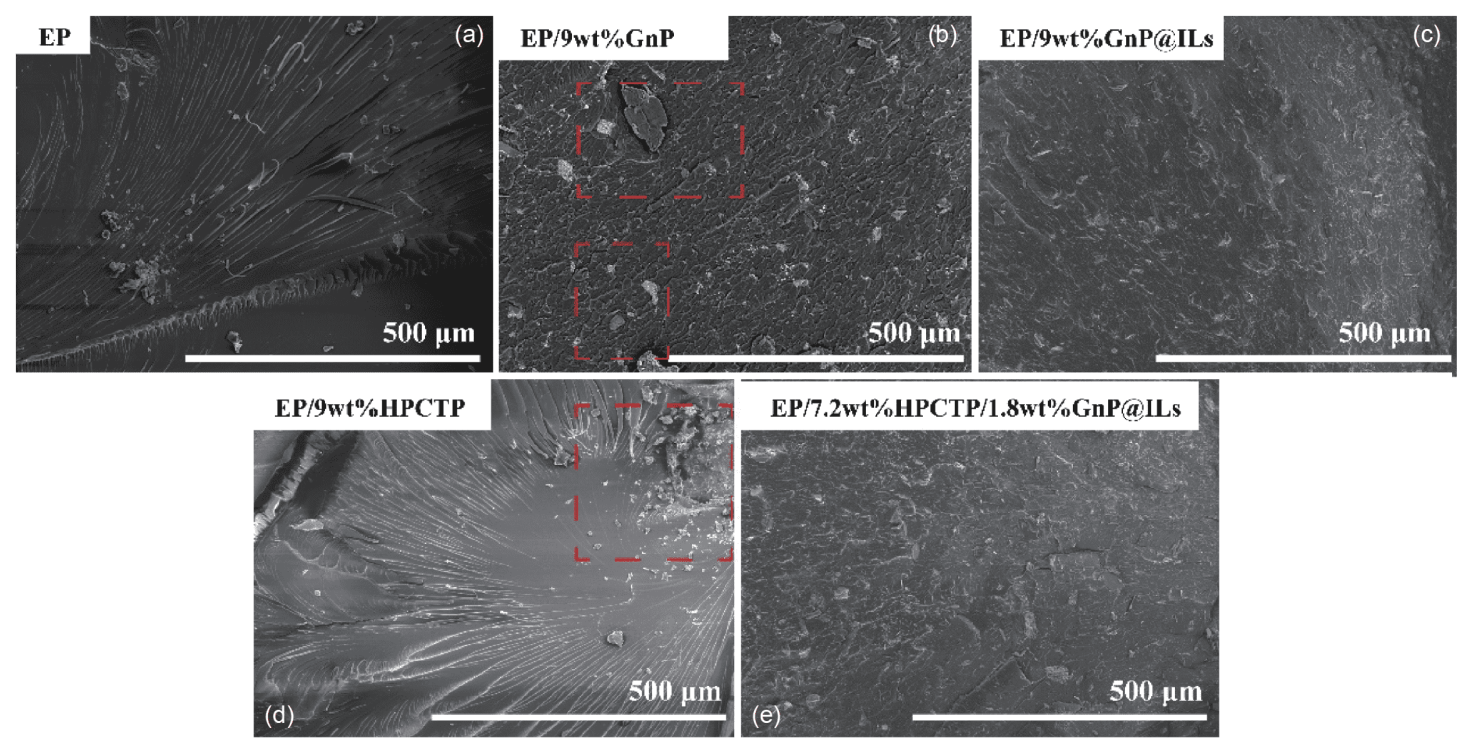

图 7 EP复合材料断面的SEM图. (a) EP; (b) EP/9wt\%GnP; (c) EP/9wt $\% \mathrm{GnP} @ I L s ;(d) \mathrm{EP} / 9 \mathrm{wt} \% \mathrm{HPCTP}$; (e) EP/7.2wt $\% \mathrm{HPCTP} /$ $1.8 \mathrm{wt} \% \mathrm{GnP} @ \mathrm{ILs}$ (网络版彩图)

Figure 7 SEM images of EP composites section. (a) EP; (b) EP/9wt\%GnP; (c) EP/9wt\%GnP@ILs; (d) EP/9wt\%HPCTP; (e) EP/7.2wt\%HPCTP/ $1.8 \mathrm{wt} \% \mathrm{GnP} @ \mathrm{ILs}$ (color online).

以上样品取平均值所得，断面样品的制备是由液氮脆 断后制备得到的. 从表中可以看出, 纯EP的TS、EB和 冲击强度分别为 $48.96 \mathrm{MPa} 、 8.96 \%$ 和 $7.49 \mathrm{~kJ} / \mathrm{m}^{2}$, 在加 入 $9 \mathrm{wt} \%$ 的 $\mathrm{GnP}$ 后力学性能明显下降, 而在加入 $9 \mathrm{wt} \%$ GnP@ILs后力学性能和纯EP相近, 这主要是由于少量 的离子液体可以增强阻燃剂与EP基体的界面相容性, 使阻燃剂均匀地分散在基体内 ${ }^{[25]}$. 同时, 在加入 $9 \mathrm{wt} \%$ 的HPCTP后, 力学性能最差, TS、EB和冲击强度分别 为 $28.16 \mathrm{MPa} 、 5.96 \%$ 和 $5.53 \mathrm{~kJ} / \mathrm{m}^{2}$, 较纯EP分别下降了 $42.49 \%$ 、33.48\%和 $26.17 \%$. 在加入 GnP@ILs后EP/ $7.2 \mathrm{wt} \% \mathrm{HPCTP} / 1.8 \mathrm{wt} \% \mathrm{GnP} @ \mathrm{ILs}$ 的力学性能有所改善, 略低于纯EP, 这归功于 GnP@ILs的增容作用 ${ }^{[25]}$.

利用SEM观察EP及其复合材料的断裂表面来分 析阻燃剂的分散情况, 如图7所示. 从图中可以看出, 纯 $\mathrm{EP}$ 表面(图7a)光滑有许多条纹. 当分别加入 $\mathrm{GnP}$ 和 HPCTP后(图7b,d), 出现了部分团聚现象, 这也解释了 其力学性能降低的原因. 而在 GnP@ILs加入后, EP $/ 9 \mathrm{wt} \%$ GnP@ILs和EP/7.2wt\%HPCTP/1.8wt\%GnP@ILs的断 面(图7c，e)由于离子液体的增容作用并没有出现明显 的团聚现象, 从而解释了其力学性能基本没变甚至有 所增加的原因.

\section{4 结论}

本文通过绿色、简单的球磨法, 成功制备出了 GnP@ILs，并利用一系列表征手段对其结构组成进行 分析. 将GnP@ILs单独或与HPCTP混合加入到EP中, 研究其对EP复合材料阻燃及力学性能的影响. 结果表 明, EP $/ 7.2 \mathrm{wt} \% \mathrm{HPCTP} / 1.8 \mathrm{wt} \% \mathrm{GnP} @ \mathrm{ILs}$ 的阻燃性能最 好, LOI值为 $33.8 \%$ 且通过了 UL-94 V 0级; 与纯EP相 比, EP/7.2wt \%HPCTP/1.8wt\%GnP@ILs的PHRR和 THR分别下降了 $55.54 \%$ 和 $44.28 \%$, TSP较EP $/ 9 \mathrm{wt} \%$ HPCTP也有所降低. 这说明了 GnP@ILs和HPCTP两者 共同作用对 $\mathrm{EP}$ 起到阻燃作用. 同时, [BMIM] $\mathrm{PF}_{6}$ 的存在 增加了阻燃剂与EP基体的相容性，使得EP复合材料的 力学性能得到了一定程度的改善.

\section{参考文献}

1 Cai H, Peng F, Wang Y, Yi J, Cai X, Liu H, Kong Q. J Nanosci Nanotechnol, 2020, 20: 6406-6412

2 Wang J. J MacroMol Sci Part B, 2020, 59: 542-550 
3 Peng HQ, Zhou Q, Wang DY, Chen L, Wang YZ. J Industrial Eng Chem, 2008, 14: 589-595

4 Liu H, Wu H, Song Q, Zhang J, Li W, Qu H. J Therm Anal Calorim, 2020, 141: 1341-1350

5 Sun F, Yu T, Hu C, Li Y. Compos Sci Tech, 2016, 136: 76-84

6 Li J, Zhao X, Wu W, Zhang Z, Xian Y, Lin Y, Lu Y, Zhang L. Curr Alzheimer Resbon, 2020, 162: 46-55

7 Wu H, Zhang L, Zhang Y, Long S, Jie X. J Alloys Compd, 2020, 834: 155107

8 Yan X, Zheng D, Yang H, Cui H, Monasterio M, Lo Y. Constr Build Mater, 2020, 257: 119477

9 Zhao Y, Liu Y, Shi T, Gu Y, Zheng B, Zhang K, Xu J, Fu Y, Shi S. Constr Build Mater, 2020, 257: 119498

10 Peng R, Wang Y, Tang W, Yang Y, Xie X. Polymers, 2013, 5: 847-872

11 Li X, Feng Y, Chen C, Ye Y, Zeng H, Qu H, Liu J, Zhou X, Long S, Xie X. J Mater Chem A, 2018, 6: 20500-20512

12 Xiao F, Wu K, Luo F, Yao S, Lv M, Zou H, Lu M. J Mater Sci, 2018, 53: 10135-10146

13 Shi YQ, Fu T, Xu YJ, Li DF, Wang XL, Wang YZ. Chem Eng J, 2018, 354: 208-219

14 Wang YH, Wu WH, Meng WH, Liu H, Yang G, Jiao YH, Xu JZ, Qu HQ. Compos Part B-Eng, 2019, 179: 107543

15 Fukunaga T, Nagano K, Mizutani U, Wakayama H, Fukushima Y. J Non-Crystalline Solids, 1998, 232-234: 416-420

16 Wang C, Wang J, Men Z, Wang Y, Han Z. Polymers, 2019, 11: 772-783

17 Huang J, Zhao X, Huang H, Wang Z, Li J, Li Z, Ji X, Cheng Y, Zhang J. Curr Alzheimer Resbon, 2019, 154: 402-409

18 Guo B, Ananth KP, Zhang J, Ji X, Bai J. Ionics, 2020, 26: 3267-3274

19 Zabihi O, Ahmadi M, Li Q, Ferdowsi MRG, Mahmoodi R, Kalali EN, Wang DY, Naebe M. J Clean Prod, 2020, 247: 119150

20 Yang Q, Zhou M, Yang M, Zhang Z, Yu J, Zhang Y, Cheng W, Li X. Nanomaterials, 2020, 10: 667-681

21 Lu S, Pei L. Int J Hydrogen Energy, 2016, 41: 15724-15732

22 Chen J, Zhu X. Food Chem, 2016, 200: 10-15

Xue BX, Niu M, Yang YZ, Bai J, Song YH, Peng Y, Liu XG. Appl Surf Sci, 2018, 435: 656-665 


\title{
The preparation of ionic liquid doped graphene composite and its synergistic flame retardant effect with hexaphenoxy cyclotripho- sphazene on epoxy resin
}

\author{
Kailun Zhang, Yiyang Chen, Meng Yao, Jiale Wang, Aiqin Wang, Hongqiang Qu*, Jianzhong Xu* \\ The Flame Retardant Material and Processing Technology Engineering Research Center of Hebei Province, College of Chemistry and Environmental \\ Science, Hebei University, Baoding 071002, China \\ *Corresponding authors (email: hqqu@163.com; xjz8112@126.com)
}

\begin{abstract}
In this article, ionic liquid doped graphene (GnP@ILs) was successfully synthesized by green and environmentally friendly wet ball milling from expandable graphite and 1-butyl-3-methylimidazole hexafluorophosphate ionic liquid $\left([\mathrm{BMIM}] \mathrm{PF}_{6}\right)$, and its surface morphology, crystal structure and chemical structure were characterized. GnP@ILs alone or mixed with hexaphenoxy cyclotriphosphazene (HPCTP) was added to epoxy resin (EP) to study its effect on the comprehensive properties of EP composites. Limiting oxygen index (LOI), vertical combustion (UL-94) and cone calorimetry test results show that GnP@ILs can improve the fire resistance of EP composites, and the EP composites (EP/7.2wt\%HPCTP/1.8wt\%GnP@ILs) has the best flame retardant performance, with an LOI of 33.8\%, and has passed the UL-94 V 0 rate. The peak heat release rate and total heat release rate of EP/ 7.2wt\%HPCTP/1.8wt\%GnP@ILs were decreased by 55.54\% and 44.28\%, respectively. At the same time, the addition of $[\mathrm{BMIM}] \mathrm{PF}_{6}$ enhanced the interface compatibility between the flame retardant and $\mathrm{EP}$, and the tensile strength and impact strength of EP composites were significantly improved.
\end{abstract}

Keywords: ionic liquid doped graphene, hexaphenoxy cyclotriphosphazene, wet ball milling, epoxy resin, flame retardant

doi: $10.1360 /$ SSC-2021-0129 